\title{
Parenteral Anticoagulation and Retroperitoneal Hemorrhage in COVID-19: Case Report of Five Patients
}

\author{
Zahra Mahboubi-Fooladi ${ }^{1}$ (D) Kowsar Pourkarim Arabi ${ }^{1}$ (D) $\cdot$ Mehdi Khazaei $^{1}$ (D) Sayyedmojtaba Nekooghadam ${ }^{2}$. \\ Bita Shadbakht ${ }^{2}$ (D) $\cdot$ Yashar Moharamzad $^{1}$ (I) $\cdot$ Morteza Sanei Taheri ${ }^{1,3}$ (I)
}

Accepted: 17 June 2021 / Published online: 26 June 2021

(C) The Author(s), under exclusive licence to Springer Nature Switzerland AG 2021

\begin{abstract}
Since coronavirus disease 2019 (COVID-19) is associated with a hypercoagulable state, especially in critical patients, anticoagulation is used for thromboprophylaxis. Hemorrhagic complications, even uncommon ones such as retroperitoneal hemorrhage, can occur following anticoagulant administration. We present 5 patients with COVID-19 whose clinical course was complicated by spontaneous retroperitoneal hemorrhage. The patients were initially presented with respiratory manifestations of the infection. There was no history or evidence suggestive for traumatic injury. After hospitalization, the patients received supplemental oxygen, antibiotics, enoxaparin or heparin, interferon beta- $1 \mathrm{~b}$ (in three patients), and anticoagulation with subcutaneous injection of enoxaparin (three patients) or heparin (two patients). During the course of hospitalization, the patients showed sudden-onset abdominal pain (three cases), hypotension (three cases), and an acute drop in hemoglobin level. CT scan of the abdomen and pelvis revealed retroperitoneal hemorrhage. For one patient, owing to unstable vital signs and an expanding hematoma, surgical intervention was performed. Others were managed conservatively with discontinuation of anticoagulants, intravenous (IV) fluid resuscitation, and packed red blood cells transfusion. Three patients died due to worsening of the infection and respiratory failure. Retroperitoneal hemorrhage could be a potential complication in COVID-19 patients receiving anticoagulation. Careful monitoring of the vital signs and blood tests like hemoglobin level of such patients is essential.
\end{abstract}

Keywords COVID-19 $\cdot$ SARS-CoV-2 $\cdot$ Hemorrhage $\cdot$ Retroperitoneal space

\section{Background}

Novel coronavirus disease 2019 (COVID-19) is a public health emergency all over the world. Many patients present with respiratory symptoms ranging from simple cough to severe shortness of breath and decreased blood oxygen levels. In addition to respiratory problems, extrapulmonary manifestations and complications of COVID-19 have been reported [1].

This article is part of the Topical Collection on COVID-19

Morteza Sanei Taheri

saneim@gmail.com

Zahra Mahboubi-Fooladi

mahboubiz@sbmu.ac.ir

Kowsar Pourkarim Arabi

medstu87@gmail.com

Mehdi Khazaei

medc2018@gmail.com

Sayyedmojtaba Nekooghadam

samonemot@sbmu.ac.ir
Severe acute respiratory syndrome coronavirus 2 (SARSCoV-2) in critically ill patients may be associated with a hypercoagulable state and subsequently result in thrombotic events in some organs such as pulmonary thromboembolism [2]. Therefore, the requirement of anticoagulant prophylaxis has been discussed in several studies [2-5]. On the other hand, various hemorrhagic conditions have been reported in COVID-19 patients. Recently, case reports have been 
published about the development of retroperitoneal hemorrhage in COVID-19 patients while receiving anticoagulation [6-8] or during extracorporeal membrane oxygenation [9].

This case series will summarize 5 proved COVID-19 patients with retroperitoneal hemorrhage while they received anticoagulant prophylaxis/treatment regarding to the COVID-19 hypercoagulable state.

\section{Case Presentation}

Here we present five patients for whom COVID-19 infection was confirmed by reverse transcriptase-polymerase chain reaction (RT-PCR) assays of the nasopharyngeal swab samples. Anticoagulation (heparin or enoxaparin) was administered for prophylaxis of thrombotic events. During admissions, they developed retroperitoneal hematomas diagnosed by CT scan imaging. The patients were hospitalized in a single teaching referral medical center. None of them had a history or evidence for traumatic injuries.

\section{Case 1}

A 65-year-old woman presented with shortness of breath and productive cough. Her past medical history included hypertension, type 2 diabetes mellitus (DM), dyslipidemia, and hypothyroidism. She was on losartan $25 \mathrm{mg}$ two times a day (BD), atorvastatin $20 \mathrm{mg}$ daily, levothyroxin $100 \mathrm{mcg}$ daily, metformin 500mg BD, and aspirin $80 \mathrm{mg}$ daily. Her symptoms had begun 4 days before her admission. Vital signs on admission showed a body temperature $(\mathrm{T})$ of $38^{\circ} \mathrm{C}$, blood pressure (BP) of 130/75 mmHg, pulse rate (PR) of $80 \mathrm{bpm}$, and respiratory rate (RR) of 22 per minute. Blood oxygen saturation $\left(\mathrm{O}_{2} \mathrm{Sat}\right)$ was $89 \%$ on room air. The initial laboratory tests revealed a hemoglobin level of $11.3 \mathrm{gr} / \mathrm{dL}$ and an elevated D-dimer level of $896 \mathrm{ng} / \mathrm{mL}$ (normal range, $<500 \mathrm{ng} / \mathrm{mL}$ ). Prothrombin time (PT), activated partial thromboplastin time (aPTT), international normalized ratio (INR), and platelet count all were within the normal laboratory range. The chest computed tomography (CT) scan showed bilateral peripheral ground glass and consolidative opacities. She was started on antibiotics (azithromycin, vancomycin, and meropenem), supplementary oxygen, and subcutaneous enoxaparin as prophylaxis (40 mg daily).

Ten days after admission, the patient's respiratory condition suddenly worsened. Therefore, with suspicion for pulmonary thromboembolism (PTE), pulmonary CT angiogram was performed. No PTE or deep vein thrombosis was documented on the pulmonary CT angiogram or ultrasound study of the lower extremities. At the same day and after a short time, she complained of a vague pain in the abdomen and on the physical examination, tachycardia and BP of $90 / 60 \mathrm{mmHg}$ were noted. Her hemoglobin level decreased to $8.4 \mathrm{gr} / \mathrm{dL}$. CT scan of the abdomen and pelvis showed a $78 \times 77 \times 75 \mathrm{~mm}$ extraperitoneal hematoma in the right hemipelvis and a $90 \times 32 \times 49 \mathrm{~mm}$ right rectus sheath hematoma (Fig. 1) Anticoagulation was discontinued immediately, and patient was resuscitated with intravenous (IV) fluid, fresh frozen plasma (FFP), and packed red blood cells (RBC). Due to the patient's unstable status and an expanding retroperitoneal hematoma on the following CT scan images, surgical intervention was performed, and $1500 \mathrm{cc}$ hematoma was removed. No active bleeding was observed during the surgery. The patient's condition gradually improved, and after 47 days of hospitalization, she was discharged.

\section{Case 2}

A 57-year-old man with a history of hypertension, type $2 \mathrm{DM}$, and chronic kidney disease (CKD) presented with myalgia, malaise, and fever for the last 4 days. He was taking losartan, atorvastatin, calcitriol, allopurinol, propranolol, nitrocontin, and glybenclamide. On admission, the patient had a body temperature of $38^{\circ} \mathrm{C}$, BP of $140 / 80 \mathrm{mmHg}$, PR of $100 \mathrm{bpm}$, RR of 20 per min, and $\mathrm{O}_{2}$ Sat of $84 \%$ on room air. His hemoglobin level was $11 \mathrm{gr} / \mathrm{dL}$, and D-dimer level was in the normal range. Chest CT scan revealed bilateral patches of ground glass and consolidative opacities suggestive for COVID-19 pneumonia. The patient was started on antibiotics (azithromycin, vancomycin, and meropenem), interferon beta- $1 \mathrm{~b}$, supplementary oxygen, hemodialysis 3 times a week, and 5000 units of subcutaneous heparin three times a day. On day 11 of the hospitalization, the patient started complaining of an abdominal pain. Physical examination showed tachycardia and hypotension $(\mathrm{BP}=90 / 70 \mathrm{mmHg})$, but the abdominal physical examination was unremarkable. Urgent laboratory work-up showed a hemoglobin level of $6.7 \mathrm{gr} / \mathrm{dL}$. The patient was transferred to the intensive care unit (ICU). Contrastenhanced CT scan of the abdomen and pelvis revealed a hematoma measuring $100 \times 100 \times 200 \mathrm{~mm}$ in the left iliopsoas muscle (Fig. 2A). Then, anticoagulation was discontinued, and the patient was treated with IV fluid, packed RBCs, and FFP. The hemoglobin level increased to $7.9 \mathrm{gr} / \mathrm{dL}$, and vital signs became stable. On the following day, her respiratory condition rapidly deteriorated, and she died at the same day due to cardiorespiratory arrest.

\section{Case 3}

An 87-year-old man with a previous history of hypertension treated with losartan $25 \mathrm{mg}$ daily presented with shortness of breath, productive cough, and decreased level of consciousness. Vital signs on admission were a body temperature of $38^{\circ} \mathrm{C}$, BP of $120 / 70 \mathrm{mmHg}$, PR of $105 \mathrm{bpm}$, and RR of $20 / \mathrm{min}$. $\mathrm{O}_{2}$ Sat on room air was $85 \%$. On the initial laboratory work-up, hemoglobin was $9.7 \mathrm{gr} / \mathrm{dL}$; PT, 
Fig. 1. Axial (A), sagittal reformatted $(\mathbf{B})$, and coronal reformatted $(\mathbf{C})$ images of the abdomen and pelvis CT scan showing a large retroperitoneal hematoma (white arrows) in the right hemipelvis that extends along the right psoas and obturator muscles. A large hematoma is noted in the right rectus abdominis muscle (arrowhead). Axial CT scan of the lung parenchyma (D) shows bilateral peripheral and peribronchovascular ground glass and consolidative opacities (black arrows).

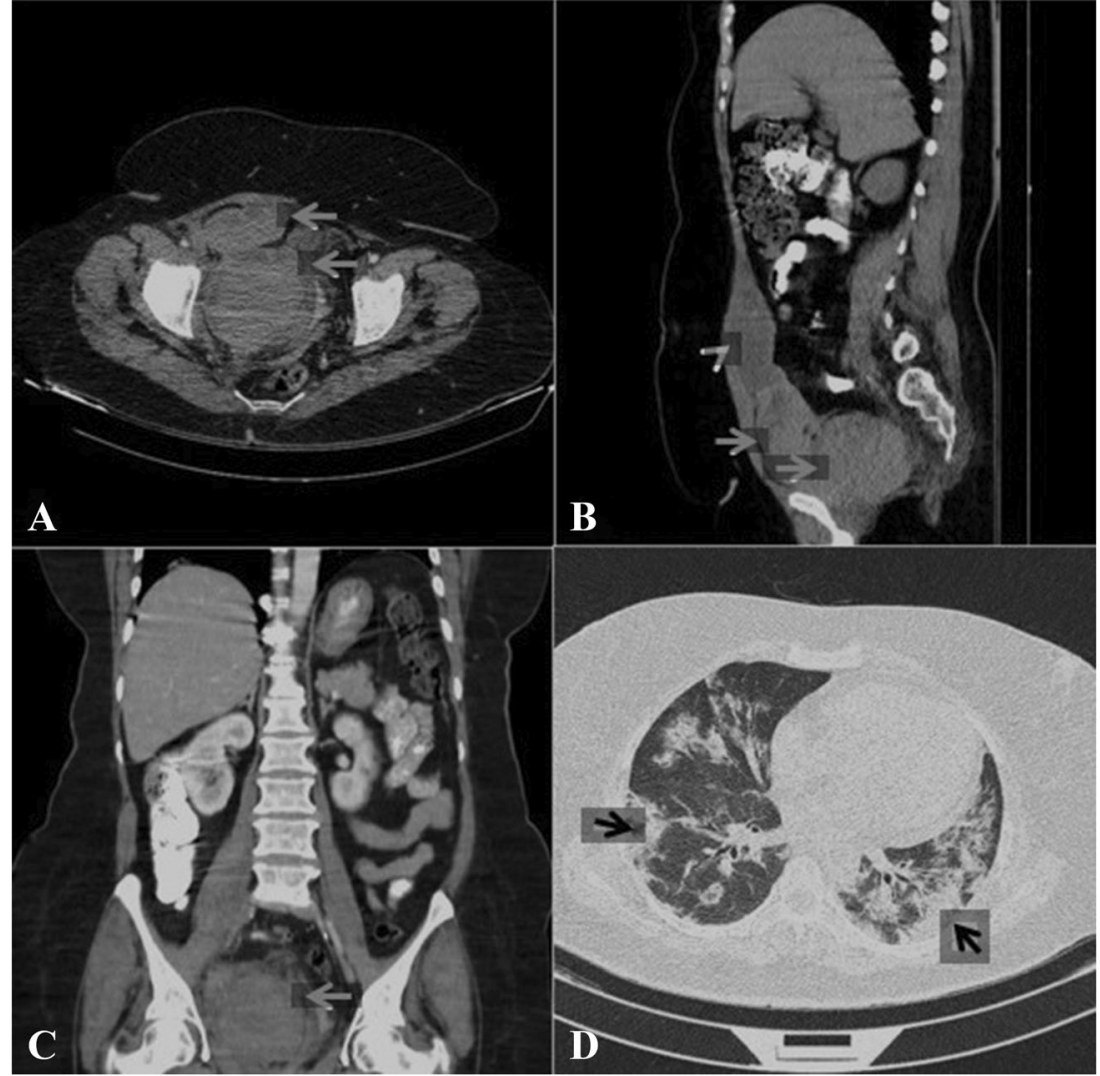

aPTT, INR, and platelet count were in normal laboratory range; and D-dimer was $1200 \mathrm{ng} / \mathrm{mL}$. Physical examination revealed pitting edema in the right lower extremity, and Doppler ultrasound demonstrated acute
Fig. 2. Retroperitoneal

hematomas on the axial CT scan of the abdomen and pelvis (white arrows) in case 2 (A), case 3 (B), case $4(\mathbf{C})$, and case 5 (D).

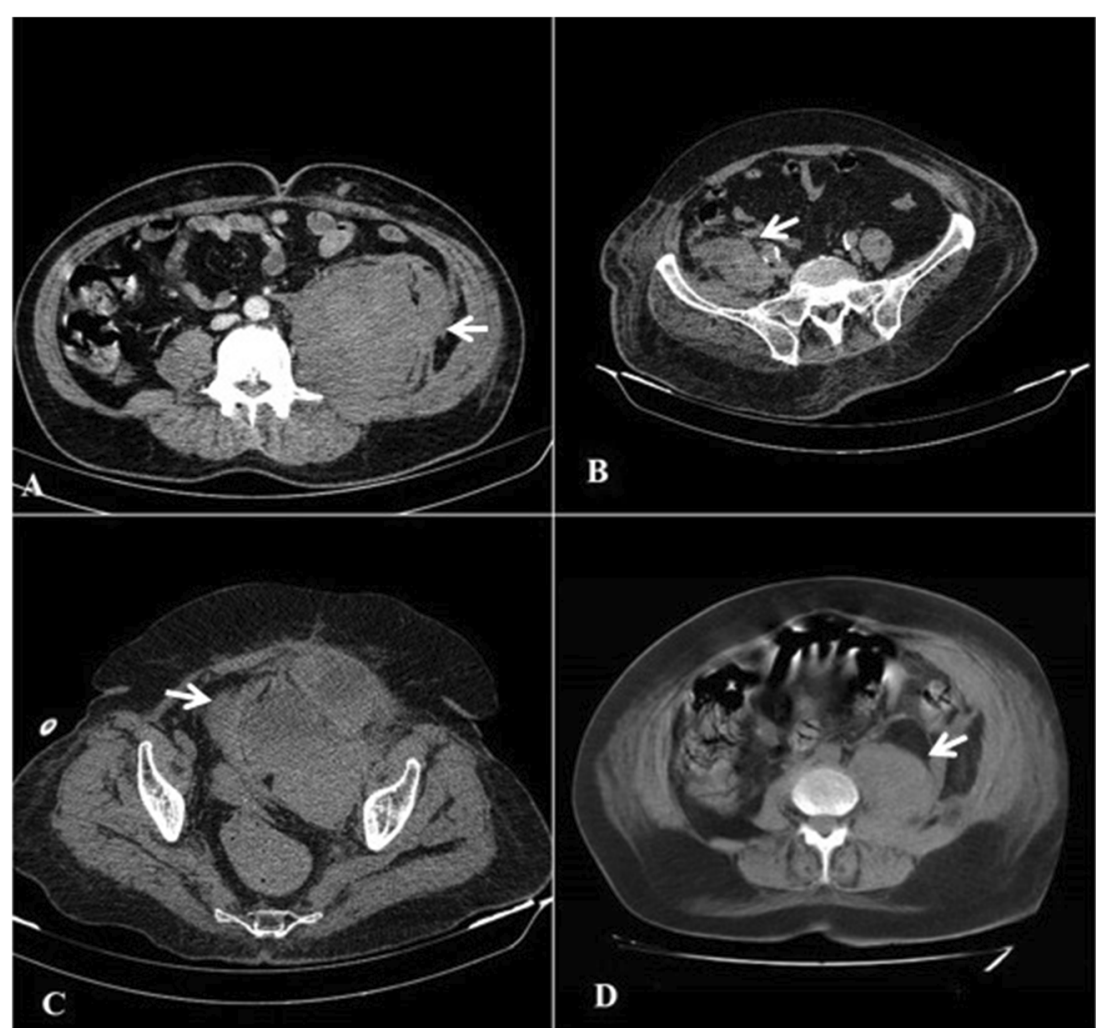

SN Comprehensive Clinical Medicine A SPRINGER NATURE journal 
thrombosis in the right common femoral vein. Chest CT scan showed peripheral patchy ground glass opacities in the right lung and complete collapse-consolidation of the left lung. Azithromycin, vancomycin, meropenem, supplementary oxygen, aspirin ( $80 \mathrm{mg}$ daily), and subcutaneous enoxaparin $(1 \mathrm{mg} / \mathrm{kg}$ twice daily) were started. On day 7 , the patient's hemoglobin dropped to $7.7 \mathrm{gr} / \mathrm{dL}$, but his BP was normal $(115 / 70 \mathrm{mmHg})$. Urgent non-contrast abdominal and pelvic CT scan revealed a retroperitoneal hematoma on the right side along the iliopsoas muscle (Fig. 2B). Anticoagulation was discontinued, and the patient was managed with IV fluid therapy and packed RBCs. The hemoglobin level increased, and vital signs became stable. The patient's respiratory condition worsened, and on the day 23 after admission, he died due to respiratory failure.

\section{Case 4}

An 81-year-old woman with a previous history of hypertension and ischemic heart disease presented with fever, malaise, productive cough, and shortness of breath. She was taking clopidogrel, losartan, and propranolol. The patient's vital signs on admission were as follows: $\mathrm{T}, 37.5^{\circ} \mathrm{C} ; \mathrm{PR}, 95 \mathrm{bpm}$; $\mathrm{RR}, 22 / \mathrm{min}$; and $\mathrm{BP}, 155 / 70 \mathrm{mmHg}$. Initially, $\mathrm{O}_{2}$ Sat with pulse oximeter was $75 \%$ under ambient air. In laboratory work-up, hemoglobin was $13 \mathrm{gr} / \mathrm{dL}$; D-dimer was $1540 \mathrm{ng} /$ $\mathrm{mL}$. Platelet count, PT, aPTT, and INR were in the normal range. Chest CT scan was performed, and bilateral diffuse ground glass and consolidative opacities were observed. The patient was started on antibiotics (azithromycin, vancomycin, and meropenem), interferon beta- $1 \mathrm{~b}$, supplementary oxygen, and 5000 units of subcutaneous heparin twice daily.

On day 9 after admission, the patient complained of abdominal pain, and her hemoglobin level dropped to $9.6 \mathrm{gr} /$ $\mathrm{dL}$. At the same time, her BP was $110 / 70 \mathrm{mmHg}$ and her PR was $90 \mathrm{bpm}$. Abdominal examination was unremarkable. The patient was admitted to the ICU. Non-contrast CT scan of abdomen and pelvis showed a $132 \times 112 \times 78 \mathrm{~mm}$ extraperitoneal hematoma in the left lower quadrant as well as left rectus sheath hematoma (Fig. 2C). Therefore, anticoagulation was stopped immediately, and IV fluid, packed RBCs, and FFP were administered. The hemoglobin level increased to $10.5 \mathrm{gr} / \mathrm{dL}$ and her vital signs normalized. Over the course of the hospitalization, the patient's clinical status and hemoglobin remained stable. She successfully recovered, and finally she was discharged on day 27 after admission.

\section{Case 5}

A previously healthy 51-year-old woman presented 1 week after initiation of dyspnea, cough, and fever. The $\mathrm{O}_{2}$ Sat on admission was $50 \%$ while breathing ambient air. The laboratory studies yielded the following values: white blood cell (WBC) count, $10,000 / \mu \mathrm{L}$ (with $30 \%$ lymphocytes and 94\% neutrophils); hemoglobin, $14.3 \mathrm{gr} / \mathrm{dL}$; and platelet count, $297,000 / \mu \mathrm{L}$. The liver function tests (LTFs) and creatinine level were within the normal limits. The patient was admitted to the ICU and received enoxaparin $(60 \mathrm{mg}$ daily), noninvasive ventilation, remdesivir, and interferon beta- $1 \mathrm{~b}$. Her respiratory condition did not improve, and on day 10 of the hospitalization, the hemoglobin level suddenly dropped to 8 $\mathrm{gr} / \mathrm{dL}$. The CT scan of the abdomen and pelvis was obtained, and a right-sided extraperitoneal hematoma within and along the iliopsoas muscle was noted (Fig. 2D). Enoxaparin was terminated, and intravenous (IV) fluid, packed RBCs, and tranexamic acid were administered. The patient's condition was stable until day 16 after admission when her respiratory status and consciousness level rapidly deteriorated. She was intubated but died on the same day due to cardiorespiratory arrest.

Table 1 summarizes clinical characteristics of the presented patients. Figures 1 and 2 show examples of retroperitoneal hemorrhages of the presented patients.

\section{Discussion}

In order to reduce thromboembolic risks and complications of COVID-19, proper treatments are necessary. However, the possibility of iatrogenic bleeding following anticoagulant administration is always a serious concern. In this report, we described five COVID-19 patients who developed anticoagulant-associated retroperitoneal hemorrhage during 7 to 11 days after admission and in-hospital treatment initiation. To the best of our knowledge, this report is the largest case series describing this complication in COVID-19 patients. Endothelial damage can explain thrombotic events seen in COVID-19. Carfora et al. suggested that this dysfunction is due to virus-assisted destruction and a general hyperinflammatory condition in the body. This causes a systemic reaction by cytokine storm which is mediated by interleukin-1 (IL-1), interleukin-6 (IL-6), tumor necrosis factor-alpha (TNF- $\alpha$ ), and abnormal complement activation.

In contrast to the increasing evidence reported in the literature regarding the procoagulability of SARS-CoV-2, hemorrhagic complications, mainly due to anticoagulation, have not been studied sufficiently in these patients [2]. Currently, clinicians have reached an agreement to use low-molecularweight heparin (LMWH) in critically ill COVID-19 patients. However, routine administration of LMWH in non-severe cases is controversial [3,4]. According to the American College of Chest Physician (CHEST) guideline, in the absence of any contraindication, it is suggested that critically ill COVID-19 patients receive thromboprophylaxis with LMWH without the requirement for routine ultrasound 
screening. In the presence of proximal DVT or pulmonary embolism, anticoagulation with LMWH or unfractionated heparin (UFH) is recommended for at least 3 months [10]. Since hematoma formation has always been known as a probable complication during LMWH treatment, clinicians should be alert about uncommon hemorrhagic complications in COVID-19 patients such as retroperitoneal hemorrhage [7].

A previous report described a case of COVID-19 with simultaneous deep vein thrombosis and retroperitoneal hemorrhage who was not receiving anticoagulation [11]. A similar case of retroperitoneal hemorrhage was reported in a patient 1 week after starting extracorporeal membrane oxygenation (ECMO) [9]. Winegarden et al. reported a COVID-19-infected female with retroperitoneal hemorrhage who presented with back and leg pain during antithrombotic treatment [12]. Another study described two patients under LMWH treatment for atrial fibrillation. These COVID-19 positive cases had spontaneous muscle hematoma [13]. Retroperitoneal hemorrhage also happened in a SARS-CoV-2 patient on daily use of aspirin for coronary artery disease and enoxaparin due to COVID-19-induced hypercoagulable state [7]. Bentivegna et al. presented a COVID-19 elderly patient with a very severe condition with multiple comorbidities like chronic atrial fibrillation who survived. They replaced her oral medication with subcutaneous LMWH for anticoagulation prophylaxis [14]. A multicenter study on 5838 patients with COVID-19 investigated results of anticoagulation treatment. This study revealed that there is no association with better outcome in coagulation prophylaxis of general population. On the other hand, those with respiratory failure and invasive ventilation had benefit [15].

It has been suggested that thromboembolic events in COVID-19 may be the result of endothelial damage [2]. On the other hand, antithrombotic and anti-platelet medications may lead to hemorrhagic complications. While the use of LMWH has been advocated in the published guidelines [10], clinicians should also be cautious about bleeding complications such as retroperitoneal hemorrhage.

\section{Conclusion}

It is well established that COVID-19 predisposes some patients to a hypercoagulable state. Hence, many clinicians favor administering anticoagulants to the admitted patients. However, this requires careful monitoring of patients during the course of receiving anticoagulants, and hemorrhagic complications, such as retroperitoneal hemorrhage, should be kept in mind. In case of rapid drop in hemoglobin level, hypotension, or back or flank pain, urgent CT scan can help in proper diagnosis and management of the patients. 
Author contribution ZMF, MKh, KPA, and MST gathered the data. $\mathrm{MKh}, \mathrm{SN}$, and BSh interpreted the data. MKh, KPA, and YM wrote the manuscript. All authors reviewed the article.

Data Availability All the required data are presented in the report.

\section{Declarations}

Ethics approval Since this was a case series and no intervention was done, ethical approval was waived.

Consent to participate Consent to participate was waived because no intervention was performed besides necessary diagnostic and therapeutic measures judged by treating physicians during admission of the patients.

Consent for publication Written informed consents were obtained from the patients or their relatives for publication.

Conflict of interest The authors declare no competing interests.

\section{References}

1. Gupta A, Madhavan MV, Sehgal K, Nair N, Mahajan S, Sehrawat TS, et al. Extrapulmonary manifestations of COVID-19. Nat Med. 2020;26(7):1017-32. https://doi.org/10.1038/s41591-020-0968-3.

2. Carfora V, Spiniello G, Ricciolino R, Di Mauro M, Migliaccio MG, Mottola FF, et al. Anticoagulant treatment in COVID-19: a narrative review. J Thromb Thrombolysis. 2020;51:1-7. https://doi.org/ 10.1007/s11239-020-02242-0.

3. Tang N, Bai H, Chen X, Gong J, Li D, Sun Z. Anticoagulant treatment is associated with decreased mortality in severe coronavirus disease 2019 patients with coagulopathy. J Thromb Haemost. 2020;18(5):1094-9. https://doi.org/10.1111/jth.14817.

4. Thachil J. The versatile heparin in COVID-19. J Thromb Haemost. 2020;18(5):1020-2. https://doi.org/10.1111/jth.14821.

5. Kow CS, Hasan SS. Use of low-molecular-weight heparin in COVID-19 patients. J Vasc Surg Venous Lymphat Disord. 2020;8(5):900-1. https://doi.org/10.1016/j.jvsv.2020.06.006.

6. Javid A, Kazemi R, Dehghani M, Bahrami SH. Catastrophic retroperitoneal hemorrhage in COVID-19 patients under anticoagulant prophylaxis. Urol Case Rep. 2021;36:101568. https://doi.org/10. 1016/j.eucr.2021.101568.
7. Patel I, Akoluk A, Douedi S, Upadhyaya V, Mazahir U, Costanzo $\mathrm{E}$, et al. Life-threatening psoas hematoma due to retroperitoneal hemorrhage in a COVID-19 patient on enoxaparin treated with arterial embolization: a case report. J Clin Med Res. 2020;12(7): 458-61. https://doi.org/10.14740/jocmr4256.

8. Scialpi M, Russo P, Piane E, Gallo E, Scalera GB. First case of retroperitoneal hematoma in COVID-19. Turk J Urol. 2020;46(5): 407-9. https://doi.org/10.5152/tud.2020.20302.

9. Guo SH, Zhu SM, Yao YX. Giant retroperitoneal hematoma during extracorporeal membrane oxygenation in a patient with coronavirus disease-2019 pneumonia. J Cardiothorac Vasc Anesth. 2020;34(10):2839-40. https://doi.org/10.1053/j.jvca.2020.05.039.

10. Moores LK, Tritschler T, Brosnahan S, Carrier M, Collen JF, Doerschug K, et al. Prevention, diagnosis, and treatment of VTE in patients with coronavirus disease 2019: CHEST Guideline and Expert Panel Report. Chest. 2020 Sep;158(3):1143-63. https://doi. org/10.1016/j.chest.2020.05.559.

11. Erdinc B, Raina JS. Spontaneous retroperitoneal bleed coincided with massive acute deep vein thrombosis as initial presentation of COVID-19. Cureus. 2020;12(8):e9772. https://doi.org/10.7759/ cureus.9772.

12. Winegarden J, Carr DB, Pike V. Severe chronic pain following retroperitoneal hemorrhage in a COVID-19 patient: amelioration with a topical pain cream. Palliat Med Rep. 2020;1(1):232-3. https://doi.org/10.1089/pmr.2020.0102.

13. Rogani S, Calsolaro V, Franchi R, Calabrese AM, Okoye C, Monzani F. Spontaneous muscle hematoma in older patients with COVID-19: two case reports and literature review. BMC Geriatr. 2020;20(1):539. https://doi.org/10.1186/s12877-020-01963-4.

14. Bentivegna E, Luciani M, Spuntarelli V, Speranza ML, Guerritore L, Sentimentale A, et al. Extremely severe case of COVID-19 pneumonia recovered despite bad prognostic indicators: a didactic report. Sn Compr Clin Med. 2020;2:1204-7. https://doi.org/10.1007/ s42399-020-00383-0.

15. Santoro F, Núñez-Gil IJ, Viana-Llamas MC, Maroun Eid C, Romero R, Fernández Rozas I, et al. Anticoagulation therapy in patients with coronavirus disease 2019: results from a multicenter international prospective registry (Health Outcome Predictive Evaluation for Corona Virus Disease 2019 [HOPE-COVID19]). Crit Care Med. 2021;49(6):e624-33. https://doi.org/10.1097/ CCM.0000000000005010

Publisher's Note Springer Nature remains neutral with regard to jurisdictional claims in published maps and institutional affiliations. 